\title{
Histopathological and molecular confirmation of Porcine Cystic Echinococcosis (CE)/Hydatidosis in Nepal
}

\author{
D. D. Joshi ${ }^{1}$, H. Yamasaki. ${ }^{2}$ \\ ${ }^{1}$ National Zoonoses and Food Hygiene Research Center (NZFHRC) Chagal, Kathmandu, Nepal ; ${ }^{2}$ Department of \\ Parasitology, National Institute of Infectious Diseases, Toyama 1-23-1 Shinjuku Ward, Tokyo 162-8640, Japan.
}

Correspondence to: Dr. Durga Datt Joshi, Executive Chairman, NZFHRC, Chagal, Kathmandu, Nepal.

Email: ddjoshi@healthnet.org.np

\begin{abstract}
Introduction: Cystic echinococcosis/hydatidosis is a cyclo-anthropozoonotic disease (parasitic infestation) of herbivorous animals and human caused by larval stage of Echinococcus tapeworm, belonging to the family Taeniidae. Dogs and some wild carnivores like foxes are definitive hosts harbouring worms in their intestine while herbivorous animals and man are intermediate host. Human acts as the dead-end host of the parasite. It is known that cystic echinococcosis in humans and pigs is prevalent in Nepal.
\end{abstract}

Methods: This study was carried out in Kathmandu valley pig rearing areas. Two cysts found in the pig pancreas were examined by histopathology and molecular analysis.

Results: In the histopathological examination, the laminated layers of the cystic walls were strongly stained with PAS, and protoscoleces were observed in the cyst. In the present study, it was first confirmed that two hydatid cysts found in pigs were identified as Echinococcus granulosus sensu stricto (sheep strain, E. granulosus G1) by mitochondrial DNA analysis.

Conclusions: Molecular analysis is indispensable not only for studying Echinococcus species associated with pathogenicity and clinical manifestation, but also for molecular epidemiology.

Keywords: Echinococcosis, Echinococcus granulosus sensu stricto, sheep strain, histopathology, mitochondrial DNA analysis

\section{Introduction}

Echinococcosis or cystic echinococcosis (CE) is caused by the larval stages (hydatid cysts) of Echinococcus granulosus and is known as one of the most important parasitic infection in livestock in the world. ${ }^{1}$ It can establish itself in many different hosts, including humans, and is regarded as one of the most widespread zoonoses. ${ }^{2}$ Domestic ungulates, including sheep, goats, cattle, swine, buffalo, horses, and camels, serve as common intermediate hosts. ${ }^{3,4}$ The definitive hosts of E. granulosus are almost invariably canid carnivores like dogs, wolves and jackals.

The concept of a E. granulosus 'strain' is developed and defined as variants 'which differ statistically from other groups of the same species in gene frequencies, and in one or more characters of actual or potential significance to the epidemiology and control of echinococcosis'.

The recognition of strain variation is a major prerequisite for control efforts aimed at limiting transmission in endemic regions of any country of the world. CE caused by $E$. granulosus continues to rank as one of the most important parasitic zoonoses worldwide, especially where there is a close association between humans and livestock. Under such circumstances, human behaviour reflected in inadequate husbandry practices is largely responsible for sustaining cycles of transmission. Education and surveillance underpin control efforts to break these cycles.

Mitochondrial DNA (mtDNA) sequences provide rich sources of data for research in evolutionary biology, 
population genetics and phylogenetics, and are increasingly being used in studies of the genus Echinococcus. To date, molecular studies, using mainly mtDNA sequences "cytochrome /c/ oxidase subunit $* 1 *(/ \operatorname{cox} / / 1 /)$ and NADH dehydrogenase subunit $1 *$ " (/nad//1/) genes, have identified nine distinct genotypes (G1-G9) within $E$. granulosus. ${ }^{6}$ In Nepal, CE is a major public health problem having been recorded in 15 districts, including villages and municipalities.

Molecular analysis showed the presence of two strains (sheep and camel) in Kenya and that the camel strain appeared restricted to the Turkana region, where camels are kept as livestock. The range of intermediate hosts for both strains appeared to be similar (sheep, cattle and camel), except that the camel strain was not isolated from any human cases examined. ${ }^{7}$ However, this strain has now been isolated from humans in Argentina, Nepal and Iran. ${ }^{8}$

Similarly in Nepal, where CE is recognized as a significant public health and environmental problem in all urban areas, three strains of E. granulosus [sheep (G1), cattle (G5) and camel (G6)] have been identified in buffalo, sheep, goat and human hosts ${ }^{9}$, of which two human isolates were identified as the camel strain (G6). There are no reports of camels being infected with E. granulosus in Nepal or Argentina; hence, the reservoir of the G6 genotype there remains undetermined although, as is the case in Argentina, goats are a likely source of infection.

The cattle adapted form has a widespread geographical distribution that includes parts of central Europe, South Africa, India, Sri Lanka, Nepal and possibly South America. ${ }^{5,10}$

\section{Methods}

Two cysts were found in the pancreas of pigs slaughtered on July 19, 2009 in Kathmandu, Nepal (Fig. 1). The cysts were approximately $2.4 \times 1.7 \mathrm{~cm}$ and $3.1 \times 2.2 \mathrm{~cm}$ in size. In the second cyst, abundant protoscoleces were visible in the hydatid fluid, but no protoscolex was observed in the smaller cyst. A part of the hydatid cysts and/or protoscoleces were fixed in 4\% paraformaldehyde and $80 \%$ ethanol for histopathology and DNA analysis, respectively.

For histopathological observation, the cyst walls were processed to paraffin-embedded specimens and thinsections were stained with PAS and observed under the microscopy. Molecular analysis was performed as follows; genomic DNA samples were prepared from ethanol-fixed cyst wall (smaller cyst) and protoscoeleces (lager cyst) using a DNeasy Blood \& Tissue Kit (Qiagen, Germany) and (coxl) was amplified by polymerase chain reaction (PCR). The PCR amplification of entire coxl was performed in a 50-mL reaction mixture with Ex Taq DNA polymerase Hot Start version (Takara Bio, Japan) and oligonucleotide primers of Eg-trnW/F (5'-GGGGCTGTTTTAGGTCATCTTATGG-3' derived from trnW gene of E. granulosus) and Eg trnT/R (5'-AGGTCAATGACTAATAATCAACTTA-3' derived from trnT gene of E. granulosus). The reaction was performed for 35 cycles of denaturation ( 94 !, $30 \mathrm{sec}$ ), annealing (58!, 30 $\mathrm{sec})$, and extension $(72$ !, $90 \mathrm{sec})$ plus one cycle of 72 ! for 5 min with a thermal cycler. Amplicons were confirmed by capillary electrophoresis (HAD-GT12, eGene Inc.) and purified using NucleoSpin Extract II kit (Macherey-Nagel, Germany) to use as templates for DNA sequencing. Samples for direct sequencing were prepared using an ABI PRISM BigDye Terminator Cycle Sequencing Ready Reaction kit (Applied Biosystems, USA) and sequencing was performed on an ABI PRISM 3100-Advant Genetic Analyzer (Applied Biosystems, USA). Sequence data were analyzed using softwares of EditSeq and MegAlign (DNASTAR Inc.) A phylogenetic tree was constructed by the neighbor-joining method using a MEGA version 4.0.

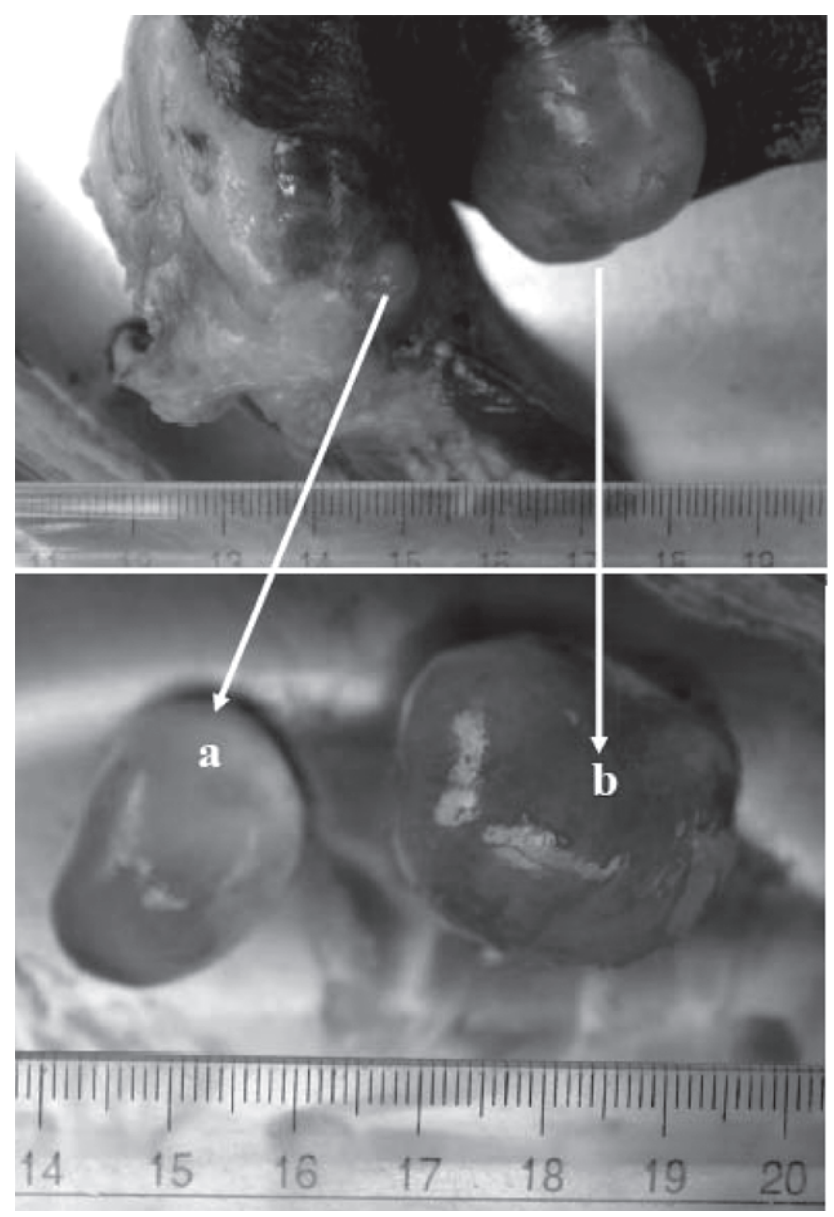

Fig. 1: Hydatid cysts found in the swine pancreas 


\section{Results}

In the histopathological examination, the laminated layers of the cystic walls were strongly stained with PAS, and protoscoleces were observed in the larger cyst (Fig. 2-B). These findings support cystic echinococcosis. Since these cysts were diagnosed as cystic echinococcosis, molecular analysis of the causative species was performed. DNA fragments $(\sim 1.8-\mathrm{kb})$ containing complete coxl genes were successfully amplified in two cases (data not shown). DNA sequence analysis revealed that a nucleotide at position 294 of the coxl was substituted ( $\mathrm{G}$ or $\mathrm{C}$ ) between two Echinococcus isolates from Nepal (AB522646 and AB522647), however both species were identified as $E$. granulosus sensu stricto (=E. granulosus, G1) (Fig. 3). It is known that cystic echinococcosis in humans and pigs is prevalent in Nepal, the infection by E. granulosus sensu stricto was first confirmed in pigs in this country.

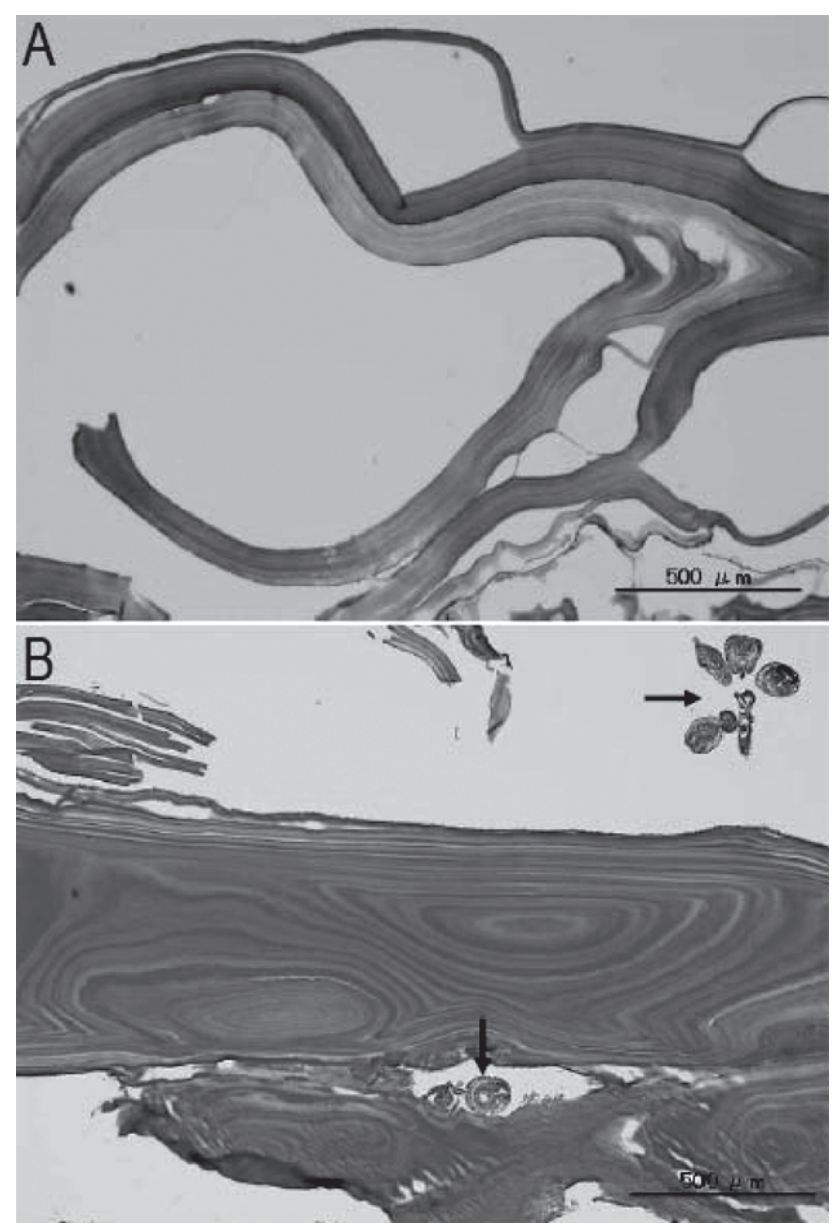

Fig. 2: Histological findings of two hydatid cysts. A, Smaller cyst ; B, Larger cyst . Note protoscoleces in the larger cyst (arrows). PAS stain.

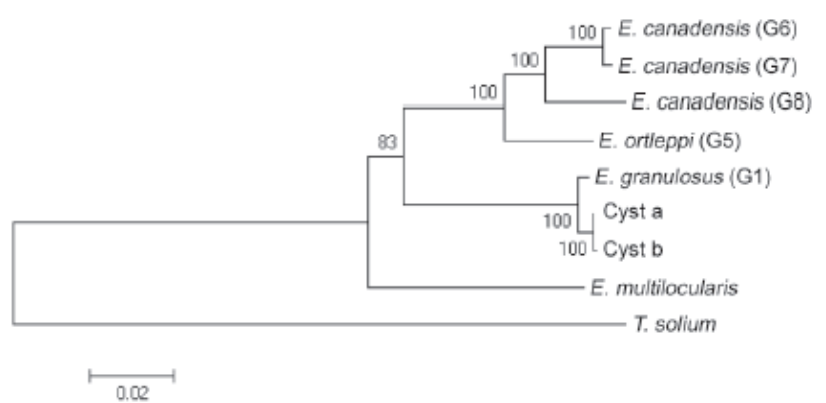

Fig. 3: Phylogenic analysis of Echinococcus species

Phylogenetic tree inferred from cox 1 gene sequences (1,609 bp) using the neighbor-joining method. DNA data are from the following accession numbers: Echinococcus granulosus (G1, AF297617), Echinococcus ortleppi (G5, AB235846), Echinococcus canadensis (G6, AB208063), E. canadensis (G7, AB235847), E. canadensis (G8, AB235848), Echinococcus multilocularis (AB018440), Taenia solium (AB086256). Numbers at branches indicate bootstrap values for 1,000 replicates and the scale bar represents the genetic distance based on Kimura's two-parameter model.

\section{Discussion}

The prevalence of echinococcosis in Nepal has been studied and observed an over all prevalence of $34.5 \%$ in buffaloes. ${ }^{11}$ It has also been reported that prevalence of echinococcosis in buffaloes was 5\% (153/3065) in Kathmandu ${ }^{12}, 21 \%{ }^{13}$, and $16.22 \%$ (73/450), in Kavrepalanchok, Nepal. ${ }^{14}$

However, the prevalence of echinococcosis has not been sufficiently examined in pigs. Humans, like other intermediate hosts becomes infected when eggs that have been shed in the faeces of the definitive host are ingested. ${ }^{15}$, ${ }^{3}$ The symptoms are determined by organ of localization, size of the cyst and their condition. ${ }^{15,3}$ The organ most commonly involved is the liver (50-70\%), followed by lungs (20-30\%) and other organs (like the spleen, kidney, heart, bones, etc.) in less than $10 \%$ of the cases. ${ }^{16}$ Mortality and case fatality rate has been recorded to be around 0.2 per 100,000 population and $2.2 \%$ respectively. ${ }^{6}$

Altogether 119 humans patents were positive for hydatid cysts out of 214,374 in-patients with a prevalence of 0.05551 $(0.056 \%$ is better) $\%$. The prevalence of hydatidosis was found higher in females as $51.26 \%$ than in males as $48.74 \%$. It has been reported $0.03 \%$ prevalence in human involving $56.7 \%$ females and $43.3 \%$ males. ${ }^{17}$ More of the patients were of the 20-49 year age category. Most of the patients with hydatid cyst belonged to the age category of 20-29 (28.57\%) with a higher prevalence in females $(64.7 \%)$ than male $(35.29 \%)$. This was because there were more female in the 
age category of $30-39$ years. ${ }^{18}$

The prevalence rate observed is higher than that observed in other studies $(8.5 \%, 12.35 \%, 5 \%, 13 \%){ }^{17,19,12,20}$ Though other studies have reported higher prevalence $(18 \%, 21 \%$, $19.5 \%) .{ }^{21,13,22}$ Bajracharya in 2009 reported the presence of the disease in lungs $(87.17 \%)$, in liver $(2.56 \%)$ and in both liver and lungs $(7.69 \%)$ and $2.56 \%$ in Spleen of buffaloes.

The overall prevalence of echinococcosis in slaughtered buffaloes in Kirtipur was to be $19.50 \%$. The prevalence was $25.00 \%$ during the winter and $14.00 \%$ during the summer. The difference was found to be significant $\left(\div^{2}{ }_{0.05}\right.$, 1d.f. $=3.851)$. Females $(23.89 \%)$ were found more infected with Echinococcus than males (13.79\%) but the difference in the sex-wise prevalence of Echinococcus was not found significant $\left(\div_{0.05}^{2}, 1\right.$ d.f. $\left.=3.19\right) .{ }^{22}$

Given the epidemiological significance of such intraspecific variation in E. granulosus and the international efforts to establish control programs in different endemic regions, an informal nomenclature was needed to reflect the phenotypic variability evident between host-derived isolates of $E$. granulosus. $^{23}$

Diagnosis of cestode zoonoses such as cysticercosis, echinococcosis, and sparganosis in humans is performed based on clinical manifestations, imaging examination, serology, and/or histopathology. PCR based mitochondrial DNA analysis using formalin-fixed paraffin-embedded sections (FPES) were highly useful for definitive diagnosis. $^{24,25}$

\section{Conclusions}

From this study it has been proved that $*^{\mathrm{m}} *$ itochondrial DNA-based analysis of cestode zoonoses has become a powerful tool not only for differential diagnosis of cystic echinococcosis from other diseases, but also for the analysis of genetic polymorphism and species identification associated with pathogenicity. This type of study should be done in Nepal in other animals in future as well which helps in differential diagnosis.

\section{Acknowledgements}

This work was supported in part by International Development Research Centre (IDRC) Ottawa, Canada and DDJ Research Foundation, Chagal, Kathmandu, Nepal and grants-in-aid from the Ministry of Health, Labour, and Welfare, Japan to HY (H20-Shinko-Ippan-016).

\section{Reference:}

1. Capuano F, Rinaldi L, Maurelli MP, Perugini AG., Veneziano V, Garippa G. Cystic echinococcosis in water buffaloes: epidemiological survey and molecular evidence of ovine (G1) and buffalo (G3) strains. Vet. Parasitol. 2006; 137: 262-268.

2. Craig PS, McManus DP, Lightowlers MW, Chabalgoity .A, Garcia HH, Gavidia C.,. Prevention and control of cystic echinococcosis. Lancet Infect. Dis. 2007; 7 : 385-394.

3. Schantz P.M. Echinococcosis. In: Steele J.H, ed. CRC Handbook Series in Zoonoses. Section C: Parasitic Zoonoses. Vol 1. Boca Raton, Florida: CRC Press: 1982; 231-277.

4. Rausch R.L. Life-cycle patterns and geographic distribution of Echinococcus species. In: Thompson R.C.A, ed. The biology of Echinococcus and hydatid diseases. London: George Allen Unwin: 1986; 44-80.

5. Thompson, R.C.A. Echinococcosis. In Principles and Practice of Clinical Parasitology, (Gillespie, S.H. and Pearson, R.D., eds), 2001; 595-612, Wiley

6. McManus DP, Zhang WB, Li J and Bartley PB. Echinococcosis. Lancet 2003; 362: 1295-1304.

7. Wachira, T.M. Bowelers, J., Zeyhel, E. \& McManus, D. P. Molecular examination of the sympatric existence and distribution of sheep and camel strains of Echinococcus granulosus in Kenya. Am. J. Trop. Med. Hyg. 1993; 48:473-479

8. Rosenzvit, M.C., Zhang, L-H, Kamenetzky, L., Canova, S.G., Guarnera, E.A. Manus, D. P. Genetic variation and epidemiology of Echinococcus granulosus in Argentina. Parasitology 1999; 118:523-530.

9. Zhang L.H., Joshi, D. D., McManus, D. P., Maharjan, M. Three genotypes of Echinococcus granulosus identified in Nepal using mitochondrial DNA markers. Trans. R. Soc. Trop. Med. Hyg. 2000; 94:258-260.

10. Thompson, R.C.A. and McManus, D.P. Aetiology: parasites and life cycles. In Manual on Echinococcosis in Humans and Animals a Public Health Problem of Global Concern (Eckert, J. et al., eds), 2001; 1-19, World Organisation for Animal Health (OIE)

11. Khan NA and Purohit SK. Prevalence of Echinococcosis in Buffaloes. Online Veterinary Journal, 2006; 1(1). www.kashvet.org/vetsscan

12. Joshi DD. Current status of Livestock Slaughtering and Meat Marketing in Kathmandu, Lalitpur and Bhaktapur. NZFHRC Kathmandu 1991; 1-162.

13. Maharjan M. Incidence of Zoonotic diseases: Echinococcosis/Hydatidosis in water buffaloes 
slaughtered for meat in western part of Kathmandu. A dissertation of M.Sc presented to Central Department of Zoology, T.U. 1996; 1-105.

14. Khatri PS. Epidemiological study of echinococcosis/ hydatidosis in human and animals of Kathmandu, Nepal. Report to IDRC, Ottawa, Canada. Study of Prevalence of Echinococcosis/Hydatidosis in different Livestock slaughtered in Banepa, Panauti and Dhulikhel Municipality of Kavrepalanchok district and its impact on Public Health. A dissertation of M.Sc presented to Central Department of Zoology, T.U.] 2003; 1-97.

15. Schantz P.M. Echinococcosis. In: Steele J.H, ed. CRC Handbook Series in Zoonoses. Section C: Parasitic Zoonoses. Vol 1. Boca Raton, Florida: CRC Press: 1982; 231-277.

16. Torgerson P.R. and Budke C.M. Echinococcosis-an international public health challenge. Res. Vet. Sci. 2003; 74: 191-202.

17. Sharma, A. 2008. Prevalence of Cystic Echinococcosis in Buffaloes Slaughtered in Kirtipur Municipality and its impact on public health. Internship Report. B.V.Sc. and A.H. 2008; 1-120.

18. Bajgain, Y.S. Assessment of public health hazard of cystic echinococcosis/hydatidosis from buffaloes slaughtered in Kathmandu and determination of critical control points. Internship report. B.V.Sc. \& A.H. 2004; $1-75$.

19. Gautam BP. Prevalence of Cystic Echinococcosis/ Hydatidosis in Slaughtered Buffaloes of Kathmandu Metropolitan City and Assessment of Its Impact on Public Health. IAAS Internship Final Report Rampur, Chitwan, Nepal. PP 2009; 1-55.

20. Joshi D.D. Veterinary Public Health Hazards in Kathmandu, Nepal. K. D. M.A. 1973; 1-45.

21. Joshi, A.B. D.D. Joshi. P.M. Schantz. H. Joshi. And A. Wald. 1996. Epidemiological assessment of echinococcosis in Nepal. In: D.D. Joshi. A.R. Walid. H. Joshi (eds.). Epidemiology of cystic echinococcosis/ hydatidosis distribution and transmission patterns in Kathmandu. Nepal. National zoonoses and food hygiene research center. Kathmandu. 1996; 81-92.

22. Bajracharya PM. Survey of Buffalo Slaughtering Places and Meat Shops of Kirtipur Municipality for Infrastructure Facilities and Prevalence of Helminth Parasites. A dissertation of M. Sc. presented to Central Department of Zoology, T.U. 2009; 1-90.
23. Yamasaki H, Nakao M, Sako Y, Nakaya K, Ito A. Molecular identification of Taenia solium cysticercus genotype in the histopathological specimens. Southeast Asian J. Trop. Med. Public Health 36 (suppl 4): 2005; S131-S134.

24. Yamasaki H, Nagase T., Kiyoshige Y. A case of intramuscular cysticercosis diagnosed definitively by mitochondrial DNA analysis of extremely calcified cysts. Parasitol. Int. 2006; 55:127-130.

25. Thompson RCA., McManus DP. Towards a taxonomic revision of the genus Echinococcus. Trend. Parasitol. 2002; 18:452-457. 